\title{
Desgaste en los sistemas de inyección diesel por efecto del elevado contenido de azufre en el petróleo diesel 2
}

\author{
Andrés Valderrama $^{\mathrm{a}^{*}}$, Jesús Flores ${ }^{\mathrm{a}}$, Manuel Bejar ${ }^{\mathrm{b}}$ y César Quispe ${ }^{\mathrm{c}}$ \\ ${ }^{a}$ Instituto de Investigación de Física, Universidad Nacional Mayor de San Marcos Ap. Postal 14-0149, Lima 14, Perú. \\ ${ }^{b}$ Departamento de Ingeniería Química, Universidad Nacional Mayor de San Marcos, Lima 14, Perú. \\ ${ }^{c}$ Departamento de Ingeniería Mecánica de Fluidos, Universidad Nacional Mayor de San Marcos, Lima 14, Perú.
}

Recibido: 11-06-2009; Aceptado: 19 -11-2009

\begin{abstract}
Resumen
El combustible empleado en las máquinas y equipos térmicos usados en las diversas actividades productivas de los sectores transporte, minería, energía, otros; es el petróleo diesel 2, que al poseer niveles altos de azufre y ejecutarse la combustión, se produce bióxido de azufre $\left(\mathrm{SO}_{2}\right)$ o partículas de sulfatos, por eso es difícil reducir la contaminación del aire generada por el uso de petróleo diesel 2, sin eliminar el azufre en su formulación; e impide el uso de muchas tecnologías convencionales y avanzadas para el control de contaminantes, incluyendo monóxido de carbono $(\mathrm{CO})$, partículas $(\mathrm{PM})$, óxidos de nitrógeno $\left(\mathrm{NO}_{\mathrm{x}}\right)$ e hidrocarburos $(\mathrm{HC})$.

En este trabajo, se comprueba experimentalmente el impacto del empleo de petróleo diesel 2, que posee elevado contenido de azufre en su formulación, sobre los componentes del sistema de inyección diesel del motor diesel turboalimentado con control electrónico Caterpillar 3412, modelo HEUI; se realiza la medición micrométrica del desgaste de la tobera del inyector y del vástago impulsor del inyector, se visualiza los vestigios de erosión y picaduras en los asientos de la válvula poppets y del inyector.
\end{abstract}

Palabras claves: Azufre; Partículas de sulfato; Bióxido de azufre; Válvula poppets

\section{Wear in diesel injection systems, the effect of the high sulfur content in diesel oil 2}

\begin{abstract}
The fuel used in thermal machines and equipment used in the various productive activities of the transport sectors, mining, energy, others are 2 diesel oil, which due to their high levels of sulfur and burning run, it produces sulfur dioxide $\left(\mathrm{SO}_{2}\right)$ and sulfate particles, so it is difficult to reduce air pollution generated by the use of petroleum diesel 2, without removing the sulfur in its formulation, and prevents the use of many conventional and advanced technologies for the control of pollutants, including Carbon monoxide (CO), particulate matter $(\mathrm{PM})$ nitrogen oxides $\left(\mathrm{NO}_{\mathrm{x}}\right)$ and hydrocarbons $(\mathrm{HC})$

In this work, we verified experimentally the impact of the use of diesel oil 2, which has high sulfur content in its formulation, the components of diesel injection system of the turbocharged diesel engine with electronically controlled Caterpillar 3412, HEUI model, is performed micrometric measurement of wear of the nozzle of the injector and injector push rod is displayed traces of erosion and pitting of the valve seats and injector poppets.
\end{abstract}

Keywords: Sulfur; Sulfate particles; Sulfur dioxide; Poppets valve.

\footnotetext{
*Contacto: avalderrama22@gmail.com
} 


\section{Introducción}

El contenido de compuestos sulfurosos en el petróleo diesel 2 (D2), se dividen en dos grupos: activos e inactivos. La existencia de azufre activo (azufre elemental(S), sulfuro de hidrogeno $\left(\mathrm{H}_{2} \mathrm{~S}\right)$ y mercaptanos (RSH)) provoca corrosión intensa en los metales del sistema de inyección en presencia de la humedad. Por ello en los D2 para motores Diesel no se admite la presencia de azufre activo, a excepción del azufre de mercaptano, cuyo contenido máximo es de $0,01 \%$. Los compuestos sulfurosos inactivos que se encuentran en el D2, durante el contacto con el metal no provocan corrosión. Sin embargo, en las condiciones de operación del sistema de inyección diesel forma anhídrido sulfuroso $\left(\mathrm{SO}_{2}\right)$ y sulfúrico $\left(\mathrm{SO}_{3}\right)$ que contribuyen el desgaste corrosivo de las piezas del sistema de inyección diesel. Es conocido y comprobado que a temperatura alta el dióxido de azufre $\left(\mathrm{SO}_{2}\right)$ y sobre todo el trióxido de azufre $\left(\mathrm{SO}_{3}\right)$ corroen los metales en la fase gaseosa y con temperatura baja, en presencia de vapores de agua, los $\mathrm{SO}_{2} \mathrm{y}$ $\mathrm{SO}_{3}$ que se forman, provocan la corrosión por ácido. La existencia de $\mathrm{SO}_{3}$ sube bruscamente el punto de rocío del vapor de agua y con esto intensifica el proceso de formación del ácido sulfúrico y acelera el desgaste de los pistones, válvulas, bujías de incandescencia y los émbolos de la bomba de inyección diesel [1].

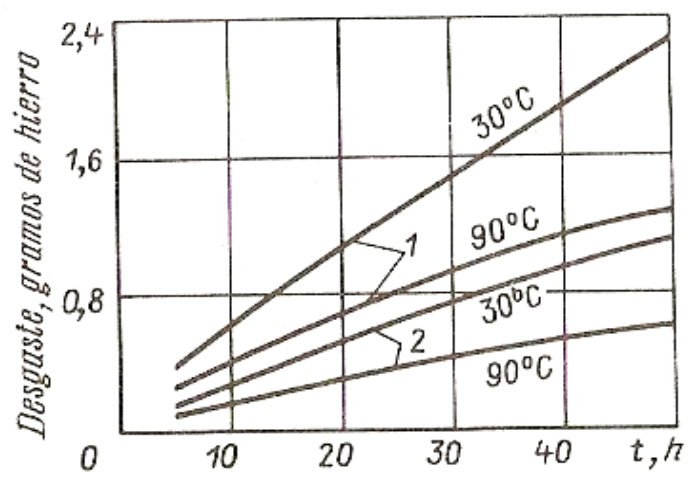

Fig.1. Influencia del \% de azufre en el desgaste del motor diesel, 1: 0,995\% y 2: 0,127\%.

El carácter ácido del desgaste corrosivo a temperatura bajas del líquido refrigerante en los motores diesel (figura 1); el ácido sulfúrico interacciona también con el aceite en la zona de los anillos, lo que conduce a la formación de compuestos químicos complejos (sedimentos carbonosos) los cuales, debido a un contenido elevado de azufre en éstos, poseen gran densidad y abrasividad [2].
El material particulado (PM) producto de la combustión del D2, son trocitos microscópicos de carbón de distintos tamaños; las más dañinas son las menores a 2.5 micras, al ser tan livianas flotan en el aire, son inhaladas y llevadas hacia los pulmones [1].

Se muestra la dependencia entre la intensidad con que se forman los sedimentos en el pistón y el desgaste de un motor diesel de un cilindro durante los ensayos realizados en 50 horas a potencia nominal y temperatura alta $\left(135^{\circ} \mathrm{C}\right)$ del líquido refrigerante en función al contenido de azufre (S) en el D2 [2].

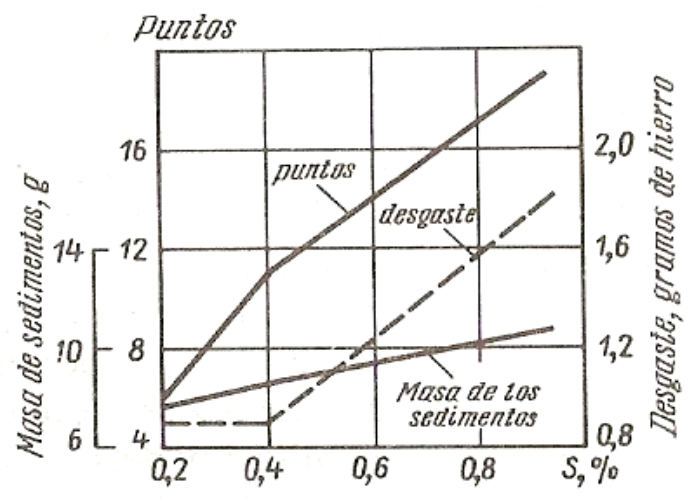

Fig. 2. Influencia sobre el desgaste y la formación de sedimentos sobre el pistón; en función del \% de azufre.

La reducción del deterioro por corrosión en los componentes del motor diesel, se logra usando aleaciones, recubrimientos anticorrosivos y agregando aditivos a los aceites lubricantes que neutralizan la acción agresiva del azufre [3].

El D2 de contenido medio de azufre ( $\sim 150 \mathrm{ppm})$ hace que la combustión sea más limpia y reduce las emisiones de PM en los motores diesel, con o sin catalizadores de oxidación. Estos beneficios se incrementan cuando los motores están diseñados para alcanzar normas de emisión más elevadas y los niveles de azufre bajan aún más [4]. Los D2 de bajo azufre ( $\sim 50 \mathrm{ppm})$ permiten mayores beneficios al incorporar tecnologías avanzadas de control; los filtros de partículas del diesel pueden usarse con combustibles de bajo azufre pero sólo alcanzan aproximadamente un $50 \%$ de eficiencia de control. La reducción catalítica selectiva puede aplicarse en este caso para lograr un control de emisiones de $\mathrm{NO}_{\mathrm{x}}$ superior al $80 \%$. Los $\mathrm{D} 2$, con ultra bajo contenido de azufre $(\sim 10 \mathrm{ppm})$ 
permiten el uso de equipo de absorción de $\mathrm{NO}_{\mathrm{x}}$, incrementando su control hasta niveles superiores al $90 \%$. Esto permite diseños de motores más eficientes, que son incompatibles con los actuales sistemas de control de emisiones [5]. Los filtros de partículas alcanzan su máxima eficiencia con combustibles de ultra bajo azufre, cerca del $100 \%$ de reducción de PM.

En el Perú, se emplea D2 con excesivo contenido de azufre (hasta 9.700 PPM), cuando lo permitido es $350 \mathrm{PPM}$, lo que origina el desgaste prematuro de los componentes del sistema de inyección diesel (válvula impelente, embolo buzo, válvula de dosificación, tobera y su asiento). Durante el proceso combustión del motor diesel se forma ácidos sulfúricos que provoca desgaste prematuro del mecanismo biela manivela (pistones, anillos, cojinetes) y desgaste del mecanismo de distribución de gases (válvulas, guías de válvulas). También, origina mayor consumo de combustible y mayor contaminación. "Los residuos de la combustión se mezclan con el lubricante de los cilindros, generando mayor desgaste del pistón, pérdida de la compresión y potencia” [3,6,11].

Los sistemas de inyección diesel con control electrónico son muy sensibles a la presencia de partículas extrañas en el D2, los motores de última generación se caracterizan por tener holguras y tolerancias muy reducidas $(\leq 2 \mu \mathrm{m})$.

El deterioro de los módulos de la bomba de inyección, se produce debido a la presencia de partículas extrañas en el D2 que obstruyen el movimiento de la válvula de dosificación de combustible generando una sobrecarga en la válvula electromagnética y como resultado circula un alto amperaje en el transistor de alta potencia para el cual el módulo no fue diseñado. Los motores diesel con control electrónico que operan en el Perú se dañan con facilidad por el alto contenido de azufre en el D2, que impide una adecuada refrigeración y lubricación de los émbolos, válvulas y toberas del sistema de inyección [6]. También, los motores diesel que poseen sistema de inyección diesel de riel común (common rail) se ven afectados y origina el reemplazo más frecuente de sus componentes.

En general, los problemas de funcionamiento por el empleo de azufre en el D2 son: disminuye la vida útil de las toberas, carbonización de las válvulas, ralentí irregular, dificultades en el arranque, altera la combustión, incremento de las emisiones, depósitos y la carbonización; se incrementa el desgaste y se origina corrosión excesiva de las tuberías de combustible, tanques de almacenamiento, bombas, otros [12].

Los límites del contenido de azufre en el D2, determinado por las normas técnicas de cada país permiten controlar el contenido de azufre en el combustible. En el Perú el D.S.025-2005PCM, establece los límites del contenido de azufre total en el D2:

Art.1 ${ }^{\circ}$. Cronograma de reducción progresiva:

\begin{tabular}{ccc}
\hline Cronograma de reducción progresiva del contenido de azufre \\
en el Combustible Diesel 1 \\
\hline Año & $\begin{array}{c}60 \text { días calendario } \\
\text { posteriores a la vigencia } \\
\text { de la presente norma }\end{array}$ & $\begin{array}{c}1 \text { de enero de } \\
2010\end{array}$ \\
Máximo azufre \\
total, \% en masa
\end{tabular}

Art. $2^{\circ}$. Prohibir la importación de D1 y D2, con concentración de azufre superiores a 2500 ppm Art $3^{\mathbf{o}}$. Posibilidad de oferta de combustible Diesel con menor contenido de Azufre.

Art $4^{\mathbf{0}}$. Incorporación de la Norma Técnica Peruana NTP321.003.2005 a las especificaciones contenidas en el Anexo A del Diesel 1, Diesel 2 (D2-350) y Diesel 2 (D2 S-50) [13].

Las anomalías por el empleo de D2, con alto contenido en azufre [2], son:

i) desgaste corrosivo en las zonas de baja temperatura de los pistones y camisas, por la formación de $\mathrm{H}_{2} \mathrm{SO}_{4}$ en las zonas subenfriadas de las camisas de cilindros [7,14]. La elevada presión de trabajo de los motores actuales facilita que se alcance el punto de rocío de las especies corrosivas que no pueden ser neutralizadas por la reserva alcalina del aceite lubricante. Por ello la utilización del D2 de bajo contenido en azufre $(\mathrm{S}<1 \%)$; significa un decrecimiento significativo de este tipo de desgaste.

ii) deposición de lacas negras en las zonas internas de las camisas de cilindros. 


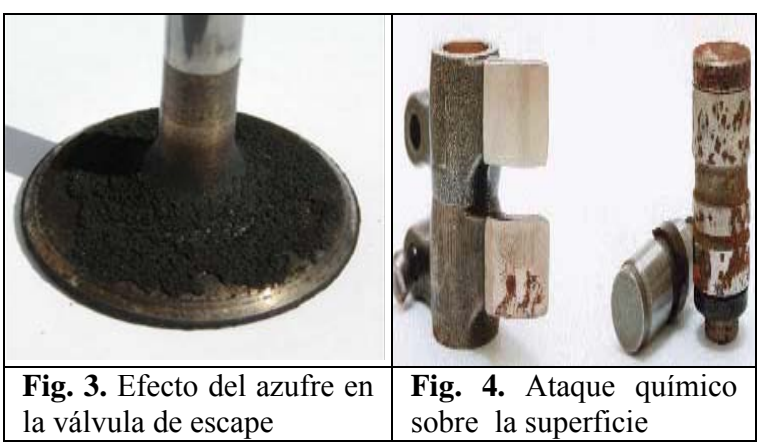

iii) desgaste corrosivo por alta temperatura en las válvulas de escape, asientos de válvulas y zonas del pistón directamente expuestas a la combustión. iv) Contaminación ambiental por la emisión de óxidos de azufre $\left(\mathrm{SO}_{\mathrm{x}}\right)$.

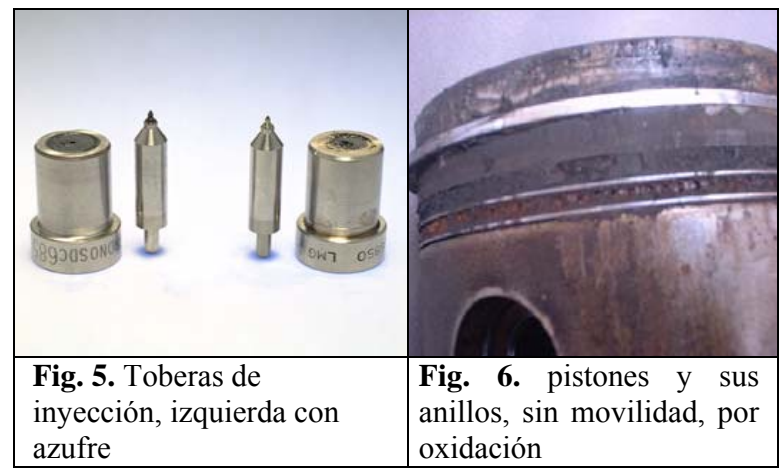

\section{Metodología Experimental}

2.1. Tractor sobre orugas, marca Caterpillar, modelo D10R, con motor diesel turboalimentado, de 14,6 litros, 4 tiempos, 12 cilindros, de inyección directa, modelo 3412 HEUI; con potencia efectiva de $525 \mathrm{~kW}$, capacidad de aceite en el cárter 37.5 galones, opera con D2.

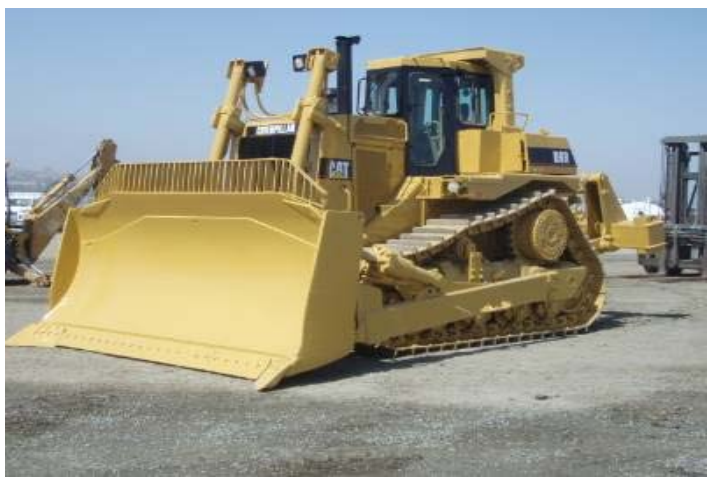

Fig. 7. Tractor de orugas CAT, D10R, de $525 \mathrm{~kW}$.

Inspección técnica de los inyectores, se desarma el inyector, se extrae la tobera con su pulverizador para el análisis correspondiente.

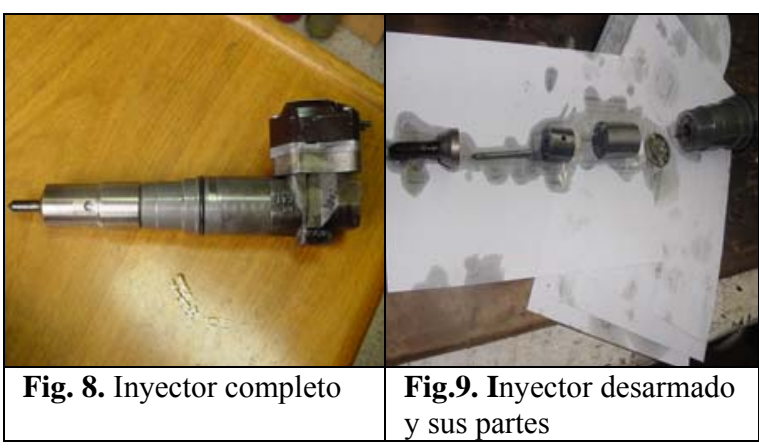

Para realizar las mediciones del diámetro del pulverizador de la tobera y del vástago impulsor del inyector, se emplearon los siguientes instrumentos:

- Micrómetro digital, Mitutoyo, con escala de 0$25 \mathrm{~mm}$, con aproximación de $0,001 \mathrm{~mm}$.

- Lupa de mecánico con aumento de 250X

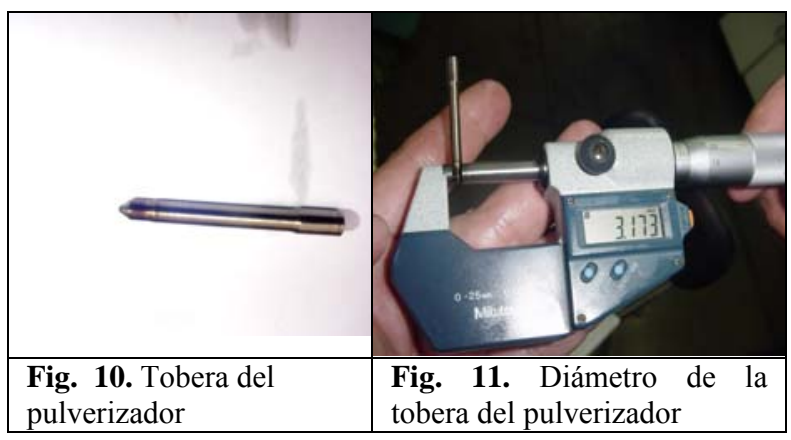

\section{Análisis de Resultados}

El análisis del desgaste de los inyectores del motor diesel turboalimentado, marca Caterpillar, modelo 3412 HEUI, instalado en el tractor de orugas modelo D10R; permite realizar el análisis de los resultados.

\subsection{Inspección del estado técnico de la} superficie exterior de los pulverizadores de los inyectores.

Los niveles de desgaste (resumen de las tablas 2 y 3), son mayores al límite máximo permisible cerca de la punta del pulverizador, como puede apreciarse en la Tabla 4.

Asimismo, se señala que en las secciones transversales I, II y III (con color negro intenso), que el desgaste que se presenta es elevado, ver figura 13.

Al introducirse el pulverizador en la tobera y ejecutar la presurización del petróleo diesel 2, se presenta una mayor holgura que la prevista, esto origina que los inyectores 1 y 2 realicen el proceso de pulverización en forma inadecuada, 
Tabla 1. Mediciones en el diámetro del vástago impulsor de los inyectores 1 y 2

\begin{tabular}{|c|c|c|c|c|c|c|}
\hline \multirow[t]{2}{*}{ Eje de medición } & \multicolumn{3}{|c|}{$\begin{array}{l}\text { Diámetro del vástago impulsor del inyector } \\
\qquad 1 \text {, en } \mathrm{mm} \text {. }\end{array}$} & \multicolumn{3}{|c|}{$\begin{array}{l}\text { Diámetro del vástago impulsor del inyector } \\
\qquad 2 \text {, en } \mathrm{mm} \text {. }\end{array}$} \\
\hline & $\mathrm{I}$ & II & III & $\mathrm{I}$ & II & III \\
\hline A-A & 3.972 & 3.968 & 3.970 & 3.968 & 3.968 & 3.969 \\
\hline B-B & 3.973 & 3.969 & 3.970 & 3.969 & 3.968 & 3.968 \\
\hline Promedio & 3.9725 & 3.9685 & 3.970 & 3.9685 & 3.968 & 3.9685 \\
\hline Diámetro estándar, mm. & 3.9750 & 3.9750 & 3.9750 & 3.9750 & 3.9750 & 3.9750 \\
\hline Desgaste, mm. & 0.0025 & 0.0065 & 0.0050 & 0.0065 & 0.007 & 0.0065 \\
\hline Desgaste límite, en mm & & 0.010 & & & 0.010 & \\
\hline
\end{tabular}

Tabla 2. Diámetro del pulverizador del inyector 1:

\begin{tabular}{|c|c|c|c|c|c|}
\hline \multirow{2}{*}{ Eje de medición } & \multicolumn{5}{|c|}{ Diámetro del pulverizador inyector 1 , en $\mathrm{mm}$. } \\
\hline & I & II & III & IV & $\mathrm{V}$ \\
\hline A & 3.173 & 3.388 & 3.391 & 3.398 & 3.997 \\
\hline $\mathrm{B}$ & 3.175 & 3.386 & 3.388 & 3.398 & 3.997 \\
\hline Promedio & 3.174 & 3.387 & 3.3895 & 3.398 & 3.997 \\
\hline Diámetro estándar, en mm & 3.200 & 3.400 & 3.400 & 3.400 & 4.000 \\
\hline Desgaste, en mm. & 0.026 & 0.013 & 0.0105 & 0.002 & 0.003 \\
\hline Desgaste límite, en mm & 0.010 & & 0.010 & & 0.010 \\
\hline
\end{tabular}

Tabla 3. Diámetro del pulverizador del inyector 2:

\begin{tabular}{cccccc}
\hline \multirow{2}{*}{ Eje de medición } & \multicolumn{5}{c}{ Diámetro del pulverizador del inyector 2, en mm. } \\
\cline { 2 - 5 } & I & II & III & IV & 3.394 \\
A & 3.175 & 3.388 & 3.389 & 3.397 & 3.993 \\
Promedio & 3.174 & 3.389 & 3.389 & 3.3955 & 3.9935 \\
Diámetro estándar, en mm & 3.1745 & 3.3885 & 3.389 & 3.400 & 0.0045 \\
Desgaste, en mm. & 3.200 & 3.400 & 3.400 & & 0.00065 \\
Desgaste límite, en mm & 0.0255 & 0.0115 & & 0.010 & 0.010 \\
\hline
\end{tabular}

Tabla 4. Desgaste en los pulverizadores 1 y 2.

\begin{tabular}{|c|c|c|c|c|c|}
\hline \multicolumn{6}{|c|}{ Inyector 1} \\
\hline Desgaste, en mm. & 0.026 & 0.013 & 0.0105 & 0.002 & 0.003 \\
\hline $\begin{array}{l}\text { Desgaste límite, en } \\
\mathrm{mm}\end{array}$ & 0.010 & & 0.010 & & 0.010 \\
\hline \multicolumn{6}{|c|}{ Inyector 2} \\
\hline Desgaste, en mm. & 0.0255 & 0.0115 & 0.011 & 0.0045 & 0.0065 \\
\hline $\begin{array}{l}\text { Desgaste límite*, en } \\
\text { mm }\end{array}$ & 0.010 & & 0.010 & & 0.010 \\
\hline
\end{tabular}

$\left(^{*}\right)$ Un consumo superior a este nivel evidencia la necesidad inmediata de reemplazo de la tobera del inyector.

Tabla 5. Desgaste en los vástagos impulsores 1 y 2.

\begin{tabular}{|c|c|c|c|c|c|c|}
\hline \multirow[b]{2}{*}{ Desgaste, $\mathrm{mm}$. } & \multicolumn{3}{|c|}{ Inyector 1} & \multicolumn{3}{|c|}{ Inyector 2} \\
\hline & 0.0025 & 0.0065 & 0.0050 & 0.0065 & 0.007 & 0.0065 \\
\hline Desgaste límite, en mm & & 0.010 & & & 0.010 & \\
\hline
\end{tabular}


Deformando el dardo de combustible, perdiendo penetración y profundidad (disminuye la capacidad de formación de la mezcla); alterando el inicio del proceso de combustión y haciendo que el motor diesel turboalimentado, marca Caterpillar, modelo 3412 HEUI, disminuya su potencia e incremente el nivel de formación y producción de hollín.

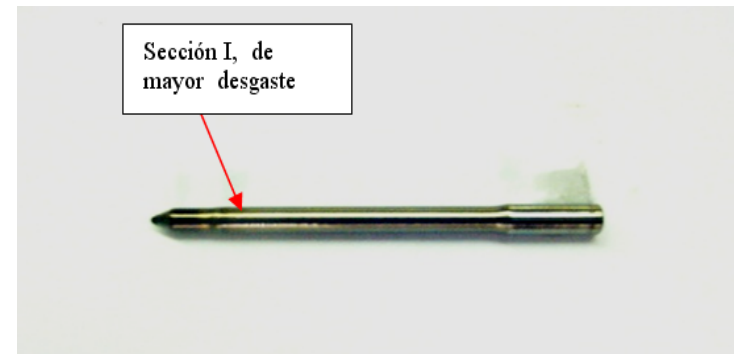

Fig. 12. Vista del pulverizador mostrando la zona I de desgaste

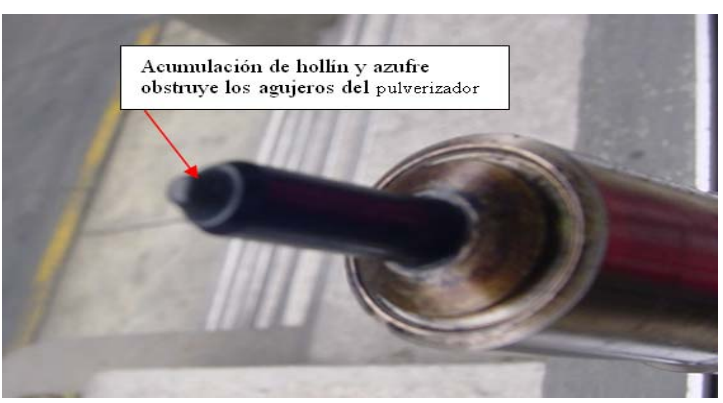

Fig. 13. Vista de los agujeros de salida del inyector

\subsection{Inspección técnica del estado técnico del diámetro del vástago impulsor del inyector.}

Durante la inspección técnica IN-SITU, se verificó que no existe desgaste excesivo; es decir, el desgaste es menor que el desgaste límite permisible, como se puede ver en la Tabla 5.

Es decir, ningún vástago impulsor posee desgaste mayor que el desgaste límite de 10 micras $(0.010 \mathrm{~mm})$, este componente podría seguir trabajando.

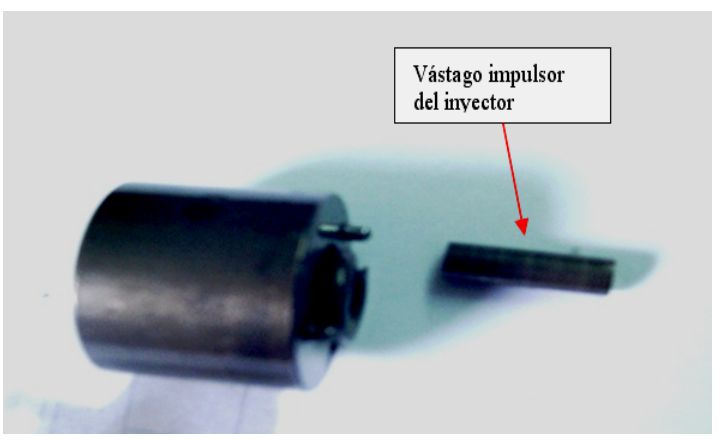

Fig. 14. Vista del vástago mostrando la zona de desgaste

\subsection{Análisis de las picaduras y erosiones en los asientos de las válvulas Poppets.}

El contenido de azufre total en el D2, realizado en CERTIPETRO-UNI, laboratorio certificado por INDECOPI:

\begin{tabular}{cc}
\hline Método de laboratorio & azufre total, ppm \\
\hline ASTM-D4294-03 & 4735 \\
\hline
\end{tabular}

De acuerdo a la NTP 321.003.2005, el límite máximo permisible es de $0,5 \%$ de azufre en masa $(5000$ ppm). Entonces, el resultado obtenido en el laboratorio es muy cercano al límite máximo permisible, pero el motor diesel CAT 3412 HEUI, es con control electrónico, sus niveles de ajuste y tolerancias es de (2-4) micras y el combustible D2, que debería emplear debe tener un nivel de contenido de azufre NO superior a $500 \mathrm{ppm}$; entonces al trabajar con un combustible petróleo diesel 2 con elevado contenido de azufre (4735 ppm), en las zonas de elevada concentración de esfuerzos mecánicos y de contacto físico entre componentes conjugados produce picaduras y erosión en las superficies de los asientos de las válvulas poppets.

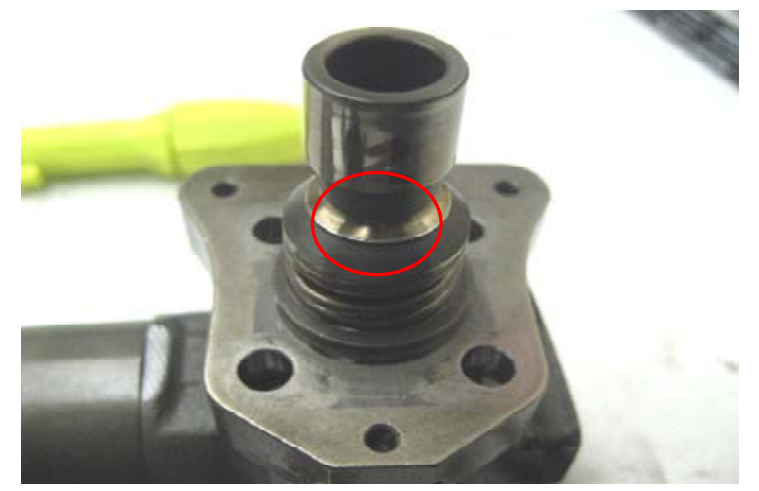

Fig. 15. Picadura y erosión en el asiento de la válvula Poppets

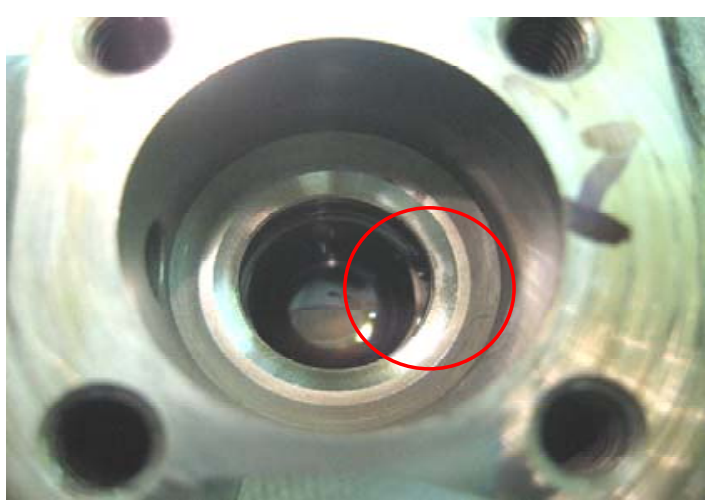

Fig. 16. Vista del inicio de erosión en el asiento, lado del inyector 
Se aprecia con nitidez el efecto del elevado contenido de azufre en el petróleo diesel 2; los vestigios de erosión en los asientos de la válvula poppets y del inyector; así como, la presencia de picaduras en el asiento de la válvula poppets.

\subsection{Contenido de metales en el D2.}

En el espectofotómetro de rayos infra-rojos, se determinó, la presencia elevada de plomo $(\mathrm{Pb})$ y de silicio ( $\mathrm{Si}$ ) (empleo de aditivos en el diesel $2)$; el contenido de fierro $(\mathrm{Fe})$, cobre $(\mathrm{Cu})$ y parte del plomo $(\mathrm{Pb})$, es producto del desgaste de los componentes del sistema de inyección diesel (toberas, pulverizadores, vástago y las válvulas Poppets), cuyo tamaño medio es inferior a las 2 micras $(<0.002)$, que atraviesa el elemento filtrante de los filtros de combustible.

Los valores de la concentración de plomo $(\mathrm{Pb})$ y fierro $(\mathrm{Fe})$ en el petróleo diesel 2, son elevados, lo que conjuntamente con el azufre y el trabajo continuo a temperatura elevada en la zona de contacto logran realizar el efecto de picadura y erosión sobre los asientos de las válvulas Poppets y de los pulverizadores de las toberas de los inyectores.

\subsection{Conteo de partículas en el D2.}

Medición de acuerdo a la norma ISO 4406, versión 1999

Tabla 7. Cantidad de partículas en el D2.

\begin{tabular}{ccc}
\hline Tamaño de partículas & $\begin{array}{c}\text { Antes del filtro, } \\
\text { PPM }\end{array}$ & $\begin{array}{c}\text { Después del filtro, } \\
\text { PPM }\end{array}$ \\
\hline CODIGO ISO, $\mu \mathrm{m}$ & $20 / 18 / 12$ & $19 / 16 / 11$ \\
\hline$>2$ & 5228 & 3666 \\
$>5$ & 1335 & 626 \\
$>10$ & 129 & 51 \\
$>15$ & 20 & 17 \\
$>20$ & 9 & 9 \\
$>25$ & 7 & 5 \\
$>50$ & 1 & 0 \\
$>100$ & 0 & 0 \\
\hline
\end{tabular}

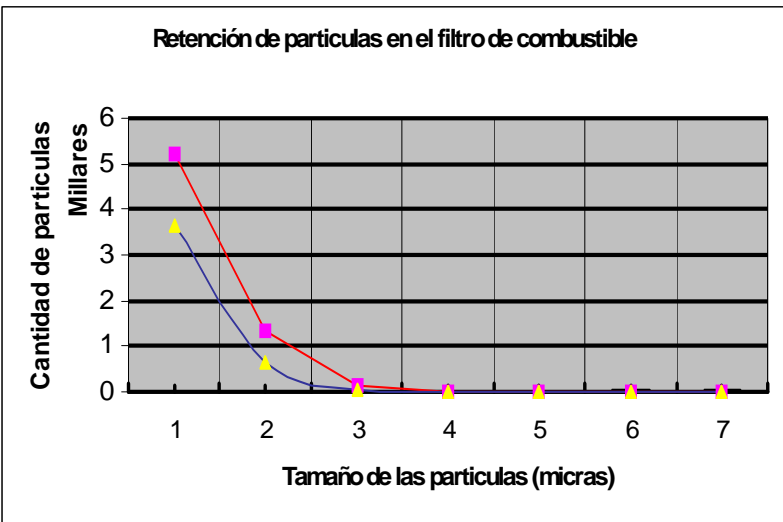

Fig. .17. Retención de partículas en el filtro para combustible: azul, filtro convencional; rojo, filtro electrónico
El trabajo del filtro para D2, se evidencia en la retención de las partículas; se puede mejorar empleando material del elemento filtrante en el rango de (2-4) micras.

\section{Conclusiones}

1. Las picaduras y erosión en los asientos de las válvulas poppets se han producido por el empleo de D2, con elevado contenido de azufre (4735 PPM). Los sistemas de inyección con control electrónico sólo permiten el empleo de combustible con 500 PPM de azufre.

2. Los pulverizadores de los inyectores y los asientos de las válvulas poppets del motor Caterpillar 3412 HEUI, poseen niveles de desgaste superiores al límite máximo permisible, originado por el elevado contenido de azufre en el D2.

3. El efecto directo del elevado contenido de azufre en el D2, se manifiesta en el contenido elevado de plomo $(\mathrm{Pb})$ y fierro $(\mathrm{Fe})$, en los vestigios de erosión en los asientos de la válvula Poppets, del inyector; en el desgaste de los pulverizadores de los inyectores y en la presencia de picaduras en el asiento de la válvula Poppets.

4. Los vástagos impulsores $\mathrm{NO}$ poseen desgaste mayor que el desgaste límite de 10 micras $(0.010 \mathrm{~mm})$, podrían seguir trabajando en los inyectores.

5. Al trabajar el motor diesel turboalimentado, marca Caterpillar, modelo 3412 HEUI, con control electrónico, empleando D2, con los pulverizadores desgastados de los inyectores, se incrementa el consumo de combustible, disminuye la potencia y se incrementa el nivel de emisiones de hollín en los gases de escape.

6. El motor diesel turboalimentado, Caterpillar, modelo 3412 HEUI, posee ajustes y tolerancias en los inyectores y componentes del sistema de inyección en el rango de (2-4) micras.

\section{Agradecimiento}

Al CSI-UNMSM que promueve las actividades de investigación, permitiendo obtener resultados concretos a los diversos problemas en el Perú. 


\section{Referencias}

[1]. Heywood, J., "Internal Combustión Engine Fundamentals", editorial McGraw Hill, pp. 491- 562, Singapore (1988).

[2]. Vsórov, B., "Manual para motores diesel para tractores", editorial MIR, pp.196-207, Moscú (1986).

[3]. Philippovich, A., "Materiales para el funcionamiento de los motores de combustión interna", editorial Labor S.A., pp. 52-67, Madrid (1944).

[4]. Regulatory Announcement: Light-Duty Diesel Tier 2- Amendments Tier 2 Vehicle \& Gasoline Sulfur Program, US EPA-2008.

[5]. Lukanin, V, "Motores de Combustión Interna", editorial MIR, pp. 153-193, Moscú (1988).

[6]. Jovaj, M., "Motores de automóvil", editorial MIR, pp. 315-381, Moscú (1982).

[7]. Bacilieva; L., "Combustibles para la operación de vehículos", editorial transport, pp. 124- 230, Moscú (1986).

[8]. Benson, R. \& Whitehouse N., "internal combustión engines", editorial Pergamon Press, pp. 145-198, Londres (1986).

[9]. Bocchi, G., "Mottori a quatttro tempi", editorial Ulrico Hoepli, pp.475-487, Milán, Italia (1988).

[10]. Ferrari, G., "Motori a combustione interna", editorial II Capitelio, pp.438-471, Torino, Italia (1992).

[11]. Obert, E., "Motores de combustión interna", editorial Continental, pp. 271-337, México (1992).

[12]. Bosch, "Manual de la Técnica del Automóvil"-2da. Edición, editorial Reverté, pp. 380- 393, Barcelona (1989).

[13]. Norma Técnica Peruana - NTP 321.003.2005, INDECOPI, Lima (2005).

[14]. Stone, R, "Introduction to internal combustion engines", editorial SAE, pp. 112-126, EE.UU. (1999). 